\title{
PHOTOMETRIC STUDIES OF Be STAR VARIABILITY
}

\author{
JOHN R. PERCY \\ Erindale Campus, University of Toronto \\ Mississauga, Ontario, Canada L5L 1 C6
}

Be stars are hot stars which have shown emission in at least one Balmer line on at least one occasion. As the definition implies, the Be phenomenon can be variable with time: on time scales of days to decades as the circumstellar disc develops and disperses; on time scales of days to months in a few Be stars which are interacting binaries; on time scales of 0.2 to 2 days due to non-radial pulsation or possibly rotation. The Be stars are worthy of photometric study because they are bright and numerous; the nature of the short-term variability is not yet agreed upon; the cause of the development of the disc - and its relationship to the short-term variability - is also not yet known.

\section{Long-Term Photometric Monitoring}

For over a decade, as part of an international UBV photometric campaign proposed and organized by $\mathrm{P}$. Harmanec and his colleagues, we have been photometrically monitoring several bright, active Be stars using two facilities: the $0.4 \mathrm{~m}$ "teaching telescope" on the main campus of the University of Toronto (Percy et al. 1988), and the $0.25 \mathrm{~m}$ Automatic Photoelectric Telescope in Arizona (Percy \& Attard 1992). The program currently includes $\theta$ CrB, 4 Her, 88 Her, 66 Oph, MWC 601, CX Dra, 2 Vul, V923 Aql, V1294 Aql, 12 Vul, 25 Cyg, 28 Cyg, QR Vul, o And, KX And and KY And. Other stars are occasionally observed as part of special projects or campaigns. Many of these stars show short-term variability; some of them (MWC 601 and QR Vul, for instance) deserve further study using a multi-longitude approach. I would be happy to send copies of the observations made since Percy and Attard's (1992), or to collaborate in multi-longitude studies of some of these stars.

\section{Short-Term Photometric Variability}

The majority of Be stars vary on time scales of 0.2 to 2 days. This variability is difficult to study because: the time scale (about a day) is very inconvenient for study from a single site; the short-term variability is not always strictly periodic; there is often additional variability from day to day because of changes in the emission or absorption by the disc; and the amplitude of the variability is a few hundredths of a magnitude at the most. We have therefore made many of our observations as part of multi-longitude "cam- 
paigns" - often as part of multi-wavelength campaigns which use optical and UV photometry and spectroscopy, along with polarimetry to understand the short-term variability (Peters et al., this meeting). The following are preliminary results of the photometric parts of some of these campaigns.

25 Cyg (HR 7647) has a suspected short period of 0.21 day (Percy et al. 1981 ), which is too short to be easily explained by rotation. From observations from Hungary, Toronto and Arizona, we have derived a "best" period of 0.226 day, with a $\mathrm{V}$ range of 0.01 , though lesser peaks are present in the power spectrum.

$\psi$ Per (HR 1087) has a suspected short-term variability of 0.05 in V (Percy et al. 1981); the period is unknown. From observations from Hungary and Arizona, we have derived a "best" period of 1.021 days. The ranges in V, B and $U$ are $0.019,0.016$ and 0.065 . The period is consistent with that derived from UV data (Peters et al., this meeting).

$\zeta$ Tau (HR 1910) has a variety of suspected short periods determined by various observers by various techniques. From observations from Hungary and Arizona, we have obtained a very complex power spectrum, with peaks around $0.3,0.67$ and 1.8 days. The period of 0.67 day fits the data well; it agrees with a period determined spectroscopically by Yang et al. (1990); and is reasonably consistent with that derived from UV data (Peters et al., this meeting). It should, however, be regarded as tentative. The ranges in $\mathrm{V}, \mathrm{B}$ and $\mathrm{U}$ are $0.071,0.064$ and 0.106 .

2 Vul (HR 7318) has a suspected period of 0.6096 day (Lynds 1959). From observations from Arizona only, we have obtained a power spectrum with the highest peak at 0.615 day. The ranges in $\mathrm{V}, \mathrm{B}$ and $\mathrm{U}$ are $0.10,0.11$ and 0.15 .

Note that, in each case, the $\mathrm{B}$ and $\mathrm{V}$ amplitudes are comparable, and the $\mathrm{U}$ amplitude is larger. The UV amplitude is larger still. This is consistent with a model in which the variability is due to temperature fluctuations in a non-radially pulsating star (Peters et al., this meeting).

Acknowledgements. I thank my campaign collaborators R.E. Fried, K. Hayhoe, I.I. Ivans, M. Paparo and S. Roy Choudhury, as well as D. Gies, H. Henrichs, D. McDavid and G. Peters for their substantial contributions to this work, and the Natural Sciences and Engineering Research Council of Canada for support.

\section{References}

Lynds, C.R. 1959, ApJ, 130, 577

Percy, J.R., Jakate, S.M. and Matthews, J.M. 1991, AJ, 86, 53

Percy, J.R., Coffin, B.L., Drukier, G.A., Ford, R.P., Plume, R., Richer, M.G. and Spalding, R. 1988, PASP 100, 1555

Percy, J.R. and Attard, A. 1992, PASP 104, 1160

Yang, S., Walker, G.A.H., Hill, G.M. and Harmanec, P. 1990 ApJS 74, 595 